\title{
Mesoporous silica nanoparticles with tunable pore size for tailored gold nanoparticles
}

\author{
G. Sponchia $\cdot$ R. Marin $\cdot$ I. Freris $\cdot$ \\ M. Marchiori • E. Moretti · L. Storaro • \\ P. Canton · A. Lausi $\cdot$ A. Benedetti $\cdot$ P. Riello
}

Received: 17 October 2013/ Accepted: 3 January 2014

(C) Springer Science+Business Media Dordrecht 2014

\begin{abstract}
The aim of this paper was to verify a possible correlation between the pore-size of mesoporous silica nanoparticles (MSNs) and the sizes of gold nanoparticles (AuNPs) obtained by an impregnation of gold(III) chloride hydrate solution in the MSNs, followed by a specific thermal treatment. Mesoporous silica nanoparticles with tunable pore diameter were synthesized via a surfactant-assisted method. Tetraethoxysilane as silica precursor, cetyltrimethylammonium bromide (CTAB) as surfactant and toluene as swelling agent were used. By varying the CTAB-toluene molar ratio, the average dimension of the pores could be tuned from 2.8 to $5.5 \mathrm{~nm}$. Successively, thiol groups were grafted on the surface of the MSNs. Finally, the thermal evolution of the gold salt, followed by "in situ" X-ray powder diffraction (XRPD) and thermogravimetric analysis (TGA),
\end{abstract}

Electronic supplementary material The online version of this article (doi:10.1007/s11051-014-2245-1) contains supplementary material, which is available to authorized users.

G. Sponchia · R. Marin · I. Freris · M. Marchiori ·

E. Moretti - L. Storaro - P. Canton - A. Benedetti .

P. Riello $(\square)$

Department of Molecular Sciences and Nanosystems,

Ca' Foscari Università di Venezia, CSGI, via Torino

155/b, 30170 Venezia-Mestre, Italy

e-mail: riellop@unive.it

A. Lausi

MCX Beamline, Elettra Sincrotrone Trieste, S.S.14-km

163.5, 34149 Basovizza, Trieste, Italy revealed an evident correlation among the degradation of the thiol groups, the pore dimension of the MSNs and the size of the AuNPs. The samples were characterized by means of nitrogen adsorptiondesorption, transmission electron microscopy, smallangle X-ray scattering, XRPD "in situ” by synchrotron radiation, and "ex situ" by conventional techniques, diffuse reflectance infrared Fourier transform spectroscopy, and TGA.

Keywords Mesoporous silica nanoparticles · Tunable pores - Surfactant-assisted method · Swelling agent . Tailored gold nanoparticles

\section{Introduction}

Silicon dioxide, especially in its amorphous form and with a nanometric scale is generating increasing interest due to its potential application as an absorbent, catalyst support and its use in biomedicine (de Dios et al. 2010; He et al. 2011a; Zong et al. 2011). It is characterized by excellent thermal and mechanical stability, good biocompatibility, and can be prepared by relatively simple synthetic procedures that allow adequate control over morphology and particle size (Lee et al. 2009; Hoshikawa et al. 2010; Lai et al. 2003). Mesoporous silica nanoparticles (MSNs) are among the most used inorganic support materials due to their large surface area and pore volume. These properties are very useful for hosting nanoparticles 
(NPs), such as therapeutic drugs and phosphors. The principal synthetic route for these nanomaterials is the mild and facile sol-gel method. Further, the in situ assembly of cetyltrimethylammonium bromide (CTAB) micellar structures in the reaction medium can allow for the synthesis of silica structures containing interconnected open pores. This encasing around these micelles by the growing silica network is the method exploited for the syntheses of the wellknown MCM-type materials (Kresge et al. 1992; Beck et al. 1992; Schumacher et al. 2000; Kim et al. 2010; Huang et al. 2010; Biz et al. 1998). Other types of nano-shaped mesoporous materials have been produced and include SBA-, MSU-, FSM-, FDU-, BMS-, and KIT-type, with morphology and pore structure being dependant on the synthetic conditions employed (Fan et al. 2003; Zhou et al. 1998; Trewyn et al. 2007; Schulz-Ekloff et al. 1999). They are characterized by a long-range order of their pores yet are mainly produced in the micrometer range (Lindlar et al. 2001). When the dimensions of the particles are reduced below the micron size, an increasing difficulty for pore size tuning and control over the particle morphology arises. Reported methods for the synthesis of MSNs using different templating agents have led to the formation of materials with pore dimensions of $4 \mathrm{~nm}$ or less (He et al. 2011b; Lin et al. 2009, 2010; Asaro et al. 2010; Cauda et al. 2010a). Such particles have attracted great interest in the biomedical field due to their inherent capability to carry within their pores a large amount of particles and ions (Enrichi et al. 2010; Parma et al. 2010; Sivestrini et al. 2010). Hence, they can be used as carriers in drug delivery acting (Huang et al. 2011; Wu et al. 2011) or as stealth NPs when properly functionalized (Park et al. 2010; Zhao et al. 2011; He et al. 2010). For such applications it may be beneficial to utilize MSNs with larger pores, combined with adequate control of the morphological shape and dimensions (Nandiyanto et al. 2009). Many other research groups have also studied the swelling properties of some aromatic compounds, including 1,3,5trimethylbenzene (TMB or mesitylene) (Fuertes et al. 2010; Galarneau et al. 2006; Lefèvre et al. 2005) or 1,3,5-triisopropylbenzene (Cao et al. 2010), or other co-solvents, for example hexane (Maldonado et al. 2007; Kruk et al. 2007; Ma et al. 2011) or $N, N$ dimethylhexadecylamine (DMHA) (Kruk et al. 2000), to enlarge CTAB micelles within the medium. Normally attention is payed only to the control of the pore enlargement of mesoporous materials, instead some authors (Büchel et al. 1998) focus also their studies to adjust the shape and size of NPs dispersed inside the MSNs. Recent decades have witnessed a tremendous interest for gold nanoparticles (AuNPs) due to their size-related properties (quantum size effect), and their wide applicability which ranges from catalysis to biology and medicine allowing, for example, heat from infrared lasers to be targeted on cancer tumors (Cai et al. 2008). The literature on this subjects is very large so, for this reason, we indicate only some reviews (Stratakis et al. 2012; Corma et al. 2008; Cai et al. 2008; Saha et al. 2012; Upadhyayula 2012). It is also well known the importance of the size of both free and supported AuNPs. Many articles discuss the role of the particle size in controlling the chemical, physical, and catalytic properties and their interaction with biological systems when the gold metal cluster measure few nanometer (Zhou et al. 2010; Scaffardi et al. 2006; Yah 2013; Cuenya 2010; Pan et al. 2007; Fenger et al. 2012; Storaro et al. 2010; Marquez et al. 2013). Preparation methods such as deposition, precipitation, co-precipitation, and impregnation have not been effective routes for supporting AuNPs on silica. The difficulty to obtain highly dispersed gold nanoparticles on silica by using these methods arises from the fact that the point of zero charge (PZC) for silica surfaces is close to $\mathrm{pH} 2$. This means that the silica surface becomes negatively charged above $\mathrm{pH} 2$, hindering the deposition of anionic gold species such as $\mathrm{AuCl}_{4}^{-}$. For this reason, when these methods are applied for gold deposition on silica, the average NPs diameter becomes very large $(>20 \mathrm{~nm})$. Giersig and Mulvaney, who showed the surface grafting of different aliphatic silanes containing terminal thiol moieties to bind gold species, first reported the stabilization of AuNPs with alkanethiols in 1993 (Giersig et al. 1993). To achieve this, a facile synthesis of thermally stable and air-stable AuNPs of reduced and controlled size by the use of thiols was described (Hasan et al. 2002). Since the capacity for controlling NPs size is one of the most important subjects of nanoscience in general, it is necessary to make nanomaterials with specific properties. Hence, the use of the mesoporous silica structure as a possible template agent for in situ controlled metal particle synthesis can be considered a useful and simple method for obtaining tailored systems. Recently, Krawiec et al. described the possibility of exploiting toluene both as a swelling agent 
and a carrier of Pt precursor inside the micelles, in order to obtain almost monodisperse metal nanoparticles inside the pores via a one step procedure (Krawiec et al. 2006). Furthermore, developments in the design of surface-functionalized MSNs have revealed the promising potential of utilizing these structurally ordered materials for therapeutic drug and/or gene delivery (Vivero-Escoto et al. 2009). MSNs offer a highly promising platform for intracellular controlled release of drugs and biomolecules. Our aim was to obtain silica nanoparticles with a spherical morphology (50-100 nm in diameter)-containing AuNPs within the pores, which are able to penetrate inside human cells and to investigate a possible correlation among the pore-size of the mesoporous silica structure and the final AuNPs grown within the silica pores (Jankiewicz et al. 2012; Choma et al. 2011, 2012). As results from the previous discussion it is evident that there is a general interest in controlling the size of the gold particles. For example the nanocomposites, developed in this study, could show good stability with respect to the sintering of the gold nanoparticles in catalytic processes, since the gold clusters are blocked inside the open pores of the MSNs. However, the present nanocomposites could be considered as a new type of material for in vivo imaging and therapy of cancer. The size of the gold particles used in such applications are usually above $20 \mathrm{~nm}$ to get a high optical activity but they cannot be easily cleared by the kidneys and therefore they are accumulated in the body. The similar optical properties could be obtained by aggregate of very closed small particles. In this case the resulting released gold NPs could be small enough to be cleared by the kidneys. In addition the studied MSNs, are also biodegradable and, with controlled size and shape (Bergman et al. 2013; Park et al. 2009; Cauda et al. 2010b). For this aim, a systematic study on the use of toluene as a swelling agent and its effect on pore size by varying its quantity have been carried out. A successive impregnation of gold salt followed by a specific thermal treatment has been considered to elucidate any possible influence of the mesoporous structure on the final nanostructure of the metal particles. Highly ordered mesoporous silica particles with sizes in the micron to sub-micron range (micron-ordered mesoporous silica particles) are of great interest due to their application as catalysts and filler materials. Alternatively, MSNs offer a highly promising platform for intracellular controlled release of drugs and biomolecules.
The study was systematically carried out as follows: (a) bare MSNs with increasing pore-size were prepared and characterized, (b) the bare MSNs were functionalized with a thiol-terminated silane (c) the functionalized MSNs were impregnated with gold(III) chloride hydrate solution (d) the evolution of the metal nanoparticles with increasing temperatures were monitored by in situ XRD measurements (d) ex situ suitable thermal treatments of the samples were performed to verify a possible correlation among pore size and AuNPs nanostructures.

\section{Experimental}

\section{Materials}

Tetraethoxysilane (TEOS, Aldrich $98 \%$ ), ethanol (EtOH, Carlo Erba 99.8 \%), ammonium hydroxide $\left(\mathrm{NH}_{3}\right.$, Fluka $\left.28 \mathrm{wt} \%\right)$, toluene (Fluka $\left.99 \%\right), n$-octane (Fluka $95 \%$ ), 1-octadecene (Aldrich $90 \%$ ), cetyltrimethylammonium bromide (CTAB, Aldrich), (3-mercaptopropyl)trimethoxysilane (MPTS, Aldrich $95 \%$ ), propylamine (Aldrich $99 \%$ ), cyclohexane (Fluka 99\%), $n$-hexane (Carlo Erba $95 \%$ ) and gold(III) chloride hydrate $\left(\mathrm{HAuCl}_{4}\right.$, Aldrich $\left.99.9 \%\right)$. Reagents were all used as received.

\section{Synthesis of the MSNs}

The synthesis of mesoporous silica nanoparticles was adapted from the procedure by Qiao et al. (2009). Deionized water $(145.2 \mathrm{~mL}, 8.07 \mathrm{~mol}), \mathrm{EtOH}$ $(22.8 \mathrm{~mL}, 0.39 \mathrm{~mol})$, and CTAB powder $(5.73 \mathrm{~g}$, $15.72 \mathrm{mmol}$ ) were stirred at $60{ }^{\circ} \mathrm{C}$. Following the complete dissolution of $\mathrm{CTAB}$, as indicated by the transparency of the solution, $1.25 \mathrm{~mL}(18.08 \mathrm{mmol})$ of a ammonia solution were added into the mixture under continued stirring and heating. After $30 \mathrm{~min}$, TEOS (14.6 mL, $64.56 \mathrm{mmol}$ ) was added dropwise. Stirring at $60{ }^{\circ} \mathrm{C}$ was continued for $2 \mathrm{~h}$, during which the clear solution gradually turned into a white suspension. The suspension was cooled to room temperature and the solid product was recovered and repeatedly washed (initially in deionized water and finally in $\mathrm{EtOH}$ ) with five cycles of centrifugation (30 min at $9 \mathrm{krpm}$ ). The dried product was finally calcined in air for $6 \mathrm{~h}$ at $550{ }^{\circ} \mathrm{C}$ in order to remove the 
Table 1 Summary of sample properties and data derived from nitrogen isotherms and TEM measurements

\begin{tabular}{llllll}
\hline Sample & $\begin{array}{l}\text { C:T } \\
\text { molar } \\
\text { ratio }\end{array}$ & $\begin{array}{l}\text { BET } \\
\text { surface } \\
\text { area } \\
\left(\mathrm{m}^{2} / \mathrm{g}\right)\end{array}$ & $\begin{array}{l}\text { BJH pore } \\
\text { diameter } \\
(\mathrm{nm})\end{array}$ & $\begin{array}{l}\text { Total } \\
\text { pore } \\
\text { volume } \\
\left(\mathrm{cm}^{3} / \mathrm{g}\right)\end{array}$ & $\begin{array}{l}\text { TEM } \\
\text { average } \\
\text { particles } \\
\text { size }(\mathrm{nm})\end{array}$ \\
\hline S & $1: 0$ & 1,000 & 2.8 & 0.94 & 75 \\
ST1 & $4: 1$ & 1,004 & 2.9 & 1.08 & 64 \\
ST2 & $1: 2$ & 997 & 3.5 & 1.85 & 55 \\
ST3 & $1: 3$ & 942 & 3.4 & 1.87 & 52 \\
ST4 & $1: 4$ & 934 & 5.5 & 2.36 & 59 \\
ST5 & $1: 5$ & 723 & 1.9 & 0.37 & \\
\hline
\end{tabular}

organic template. All samples details and labels are reported in Table 1.

AuNPs growth into silica network

Thiol-functionalization was made before impregnating silica with gold salt solution. Adaptation of the method performed by Chen et al. for surface grafting of functionalized silane was used (Chen et al. 1982). Similarly to the literature, the mercaptopropyl-functionalised MSNs was prepared using a cyclohexane $(5 \mathrm{~mL})$ solution-containing $2 \% \mathrm{v} / \mathrm{v}$ MPTS and $2 \% \mathrm{v} / \mathrm{v}$ $n$-propylamine. Thiol-functionalized MSNs (100 mg) was placed in a round-bottom flask equipped with magnetic stirrer. Ethanol and $25 \mathrm{mM} \mathrm{HAuCl}_{4}$ solution were added to the powder, in order to introduce approximately $8 \mathrm{wt} \%$ of gold in the materials, while stirring under nitrogen. After $30 \mathrm{~min}$ the solvent was removed under reduced pressure $\left(10^{-1} \mathrm{mbar}\right)$ by gentle heating in a water bath $\left(60^{\circ} \mathrm{C}\right)$.

\section{Characterization}

The nitrogen adsorption-desorption isotherms were measured at liquid nitrogen temperature $\left(-196{ }^{\circ} \mathrm{C}\right)$ using a Micromeritics ASAP 2010 volumetric adsorption analyzer. The Brunauer-Emmett-Teller (BET) equation was used to calculate the specific surface area from adsorption data and the pore-size distributions were calculated from the adsorption branches of the isotherms based on the Barrett-Joyner-Halenda (BJH) model. The dimensions and the morphologies of the nanoparticles were studied by transmission electron microscopy (TEM) using a JEOL JEM 3010 operating at $300 \mathrm{kV}$. The energy dispersive $\mathrm{X}$-ray spectroscopy
(EDS) were collected with JEOL JSM-5600LV scanning electron microscope (SEM). The IR spectra were recorded with a NEXUS-FT-IR instrument implementing a Nicolet AVATAR Diffuse Reflectance accessory. The thermogravimetric analyses were performed in air from 30 to $900{ }^{\circ} \mathrm{C}$ with a heating rate of $20^{\circ} \mathrm{C} / \mathrm{min}$ with a Netzsch STA 409. The diffraction patterns were recorded with a small-angle X-ray scattering (SAXS) Kratky camera $\left(\mathrm{Cu} K \alpha_{1}=0.154 \mathrm{~nm}\right)$ equipped with a proportional detector for intensity collection. The sample was held in a 1-mm thick sample holder at room temperature. Data were acquired as a function of the scattering vector modulus $h=(4 \pi \sin \theta) / \lambda$ where $2 \theta$ is the angle between the incident and scattered beams. The SAXS curves were obtained by step scanning in the range from 0.01 to $0.4 \AA^{-1}$. The temperature wideangle X-ray scattering (WAXS) measurements were collected at the ELETTRA Laboratories of Sincrotrone Trieste S.C.p.A (Line MCX) using a quartz glass capillary as sample holder (Riello et al. 2013). A Philips X'Pert vertical goniometer with Bragg-Brentano geometry, connected to a highly stabilized generator, was used for all the other X-ray powder diffraction (XRPD) measurements; a focusing graphite monochromator and a proportional counter with a pulseheight discriminator were used, Nickel-filtered $\mathrm{CuK} \alpha$ radiation and a step-by-step technique were employed (steps of $0.05^{\circ} 2 \mathrm{~h}$ ), with collection times of $10 \mathrm{~s} / \mathrm{step}$. These latter patterns were used for the quantitative analysis and size distributions of AuNPs using the Rietveld method (Riello et al. 1998a).

\section{Results and discussion}

\section{Pore-expanded MSNs}

Hydrophobic solvents as toluene, $n$-octane and 1-octadecene were selected as suitable swelling agents. Several samples having a different molar ratio between the surfactant $(C)$, and the swelling agent $(T)$ were synthesized. It was found that both 1-octadecene and $n$-octane were not suitable since they gave rise to phase separation and to no noticeable increase in the pore size. These data are reported in Fig. S1 in supporting Information (SI). Instead, an homogenous phase was observed in the toluene-containing system for all the different molar ratios investigated. The resulting mesoporous materials were characterized by 
the formation of pores with controlled size distribution in a disorder structure. This evidence suggests that the reaction conditions and molar ratio are suitable for the synthesis of our mesoporous materials. Similar results are present in the literature where some authors (Lindén et al. 2000; Ottaviani et al. 2004; Poteshnova et al. 2006; Wan et al. 2007; Firouzi et al. 1995; Biz et al. 1998) report the detailed phase diagrams for systems resembling the one we have studied, however a specific phase diagram for the toluene system is not reported. Looking at the molar ratio of the reagents used in the cited literature and considering also that the increasing of organic co-solvent results in a final structure which is less ordered and less expanded, it is possible to obtain some good starting values for our system to accomplish micellar system apt to our final goal. For all these reasons there should not be any hindrances for this system to produce a final material having all the characteristics necessary for our final applications. On this basis, all the subsequent experiments were performed using toluene as swelling agent. The textural properties of the MSNs prepared with the different CTAB:toluene (C:T) molar ratios are listed in Table 1.

Thermogravimetric analysis (TGA) was carried out to find the optimal post-synthesis thermal treatment so that to maintain the reactive $\mathrm{Si}-\mathrm{OH}$ groups on the surface and to assure the complete removal of the organic precursor which renders the MSNs unsuitable for biological applications. In previous studies it was found that a thermal treatment at $550{ }^{\circ} \mathrm{C}$ for $6 \mathrm{~h}$ was sufficient to remove the surfactant molecules without complete dehydroxylating the superficial - $\mathrm{OH}$ groups and without collapsing the pore network (Parma et al. 2010). To check for the applicability of the procedure a TGA was performed on sample ST4. Figure S2 shows that the majority of weight loss occurs before reaching $400{ }^{\circ} \mathrm{C}$. An initial loss of $5 \%$ was due to the removal of residual solvent. A second minimum at $250{ }^{\circ} \mathrm{C}$, visible in the derivative of thermogravimetric curve (DTG) was ascribed to the removal of the organic fraction comprising CTAB which has a melting point of $237{ }^{\circ} \mathrm{C}$ and decomposes at $243{ }^{\circ} \mathrm{C}$. The differences between the temperatures reported in the TGA with the literature values, could be due to the CTAB encapsulation in the pore which creates an interaction between the surfactant molecules and the internal surface of the MSNs. The combustion of CTAB from within the pores requires higher energies and temperatures than the ones needed for its bulk decomposition. In the $250-550{ }^{\circ} \mathrm{C}$ temperature range the combustion of unreacted ethoxy groups arising from TEOS, the decomposition of residual organic molecules and the condensation of neighboring silanol groups occurred. Above $550{ }^{\circ} \mathrm{C}$ a further loss of approximately $2 \%$ was observed due to thermal dehydroxylation of internal surface silanol groups to form siloxane bridges.

To confirm the absence of the organic fraction and the presence of superficial-free silanol groups, the DRIFT-IR spectra of the ST4 sample as-prepared and after calcination at $550{ }^{\circ} \mathrm{C}$ were compared (Fig. S3). The broad absorption band arising from the vibrational modes of the water was strongly reduced in the sample annealed at $550{ }^{\circ} \mathrm{C}$; in the proximity of this region the appearance of a sharp peak at $3,746 \mathrm{~cm}^{-1}$ was attributed to the stretching of the superficial silanol groups. The presence of these reactive superficial groups was the most important objective of post-synthesis calcination together with the disappearance of the features under $3,000 \mathrm{~cm}^{-1}$ and the peak at $1,480 \mathrm{~cm}^{-1}$ arising, respectively, from the vibrational modes of aliphatic groups and from the in plane bending of aliphatic groups, which confirms the removal of the organic fraction (Bae et al. 2006). The characteristic absorptions of the silica were present in both spectra: in particular the very strong bands between 1,400 and $1,000 \mathrm{~cm}^{-1}$ are ascribed to the symmetric stretching of siloxanic groups (Badaničová et al. 2010). The presence of a stronger band of the absorbed water at $1,628 \mathrm{~cm}^{-1}$ in the treated sample can be attributed to humidity adsorption from the atmosphere.

In order to study the swelling effect of the toluene, nitrogen physisorption analyses were made for each prepared sample. The $\mathrm{N}_{2}$ absorption-desorption hysteresis curves (Fig. 1) showed a progressive increase in the volume of the adsorbed gas with the increasing in toluene concentration, maintaining a type IV shape. When the molar ratio $\mathrm{C}: \mathrm{T}$ is $1: 5$, the curve assumed a different profile, displaying a type I isotherm (Fig. 1). This suggests a collapse of the micellar structure and the observed porosity can be attributed to micropores. It is likely that the micelles have swelled to the extent to which they break; the porosity of the sample arises from the presence of randomly distributed organic fraction. The BET surface areas, the total pore volume and the average pore diameters, evaluated by $\mathrm{BJH}$ method (Fig. 2), are summarized in Table 1. 

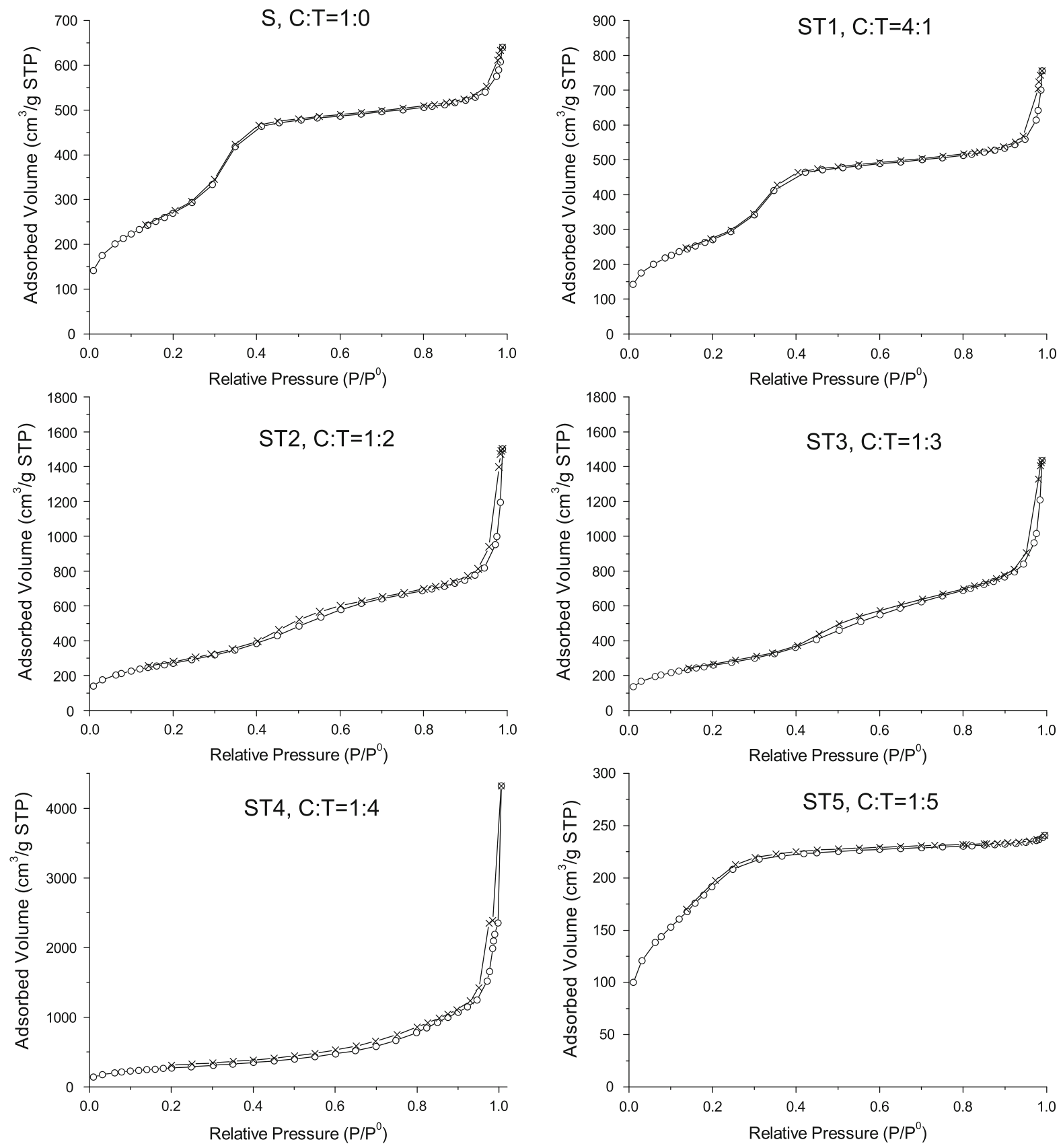

Fig. 1 Adsorption-desorption curves of the samples S, ST1-5. The curves are of type IV of the IUPAC classification of 1984, except for the sample ST5 which shows a type I curve. The

The decrease of the CTAB-toluene molar ratio led to a growth in the pore size and in the total pore volume, until the aforementioned critical ratio 1:5 was reached. It is worth noting that as the mean pore size increases the distribution is broadened towards larger

volume of adsorbate increases monotonically with the increase of the toluene content, and has an abrupt fall when the molar ratio between $\mathrm{CTAB}$ and the aromatic solvent reaches 1:5

diameters. The largest pore diameter was observed with a toluene concentration four times higher than the surfactant. In this sample (ST4) the surface area maintains appreciable values, the mean pore size is centered at around $5.5 \mathrm{~nm}$, and the total pore volume is 


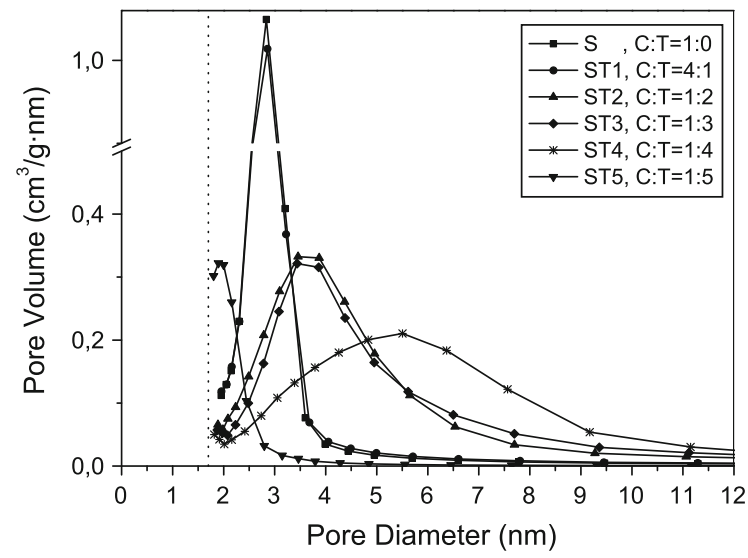

Fig. 2 Pore diameter distribution, calculated according to the BJH approach, of the sample synthesized without the addition of toluene (S) and for the samples prepared with decreasing CTAB-toluene molar ratio (ST1-ST5)

quite high. The shift of the intermediate step in the isotherms points out the growth of pore dimensions. Furthermore, the isotherms flattening in samples with lower C:T molar ratio highlights a polydispersion of channel diameters. A further increase in the concentration of the swelling agent, sample ST5, caused the collapse of the template structure and led to microporosity, reduced surface area, and total pore volume.

A parallel study of the materials was carried out using the SAXS technique. The low angle region of the spectrum provides information on the arrangement and morphology of the pores. The spectra of the samples are reported in Fig. S4 where the peaks arising from the interference due to the pore ordering can be appreciated ( $\mathrm{Li}$ et al. 2001). The shift toward lower $h$ values and the broadening of the peaks by increasing the quantity of toluene was consistent with the increase in the pore diameter and the broadening of their distribution, so that the channels tended to be arranged in a more random fashion.

Finally, the samples were examined by TEM (Figs. 3, S5 in SI). The micrographs of Fig. 3 depict an evident increase in the porosity in correspondence to the decreasing C:T molar ratio from sample $\mathrm{S}$ to sample ST4. All these samples showed a random distribution of the pore channels. Mesoporous materials are rather disordered in presence of swelling agent in agreement with the literature (Wan et al. 2007). The enlarging of the pores led to a reduction in the thickness of the silica walls, which became
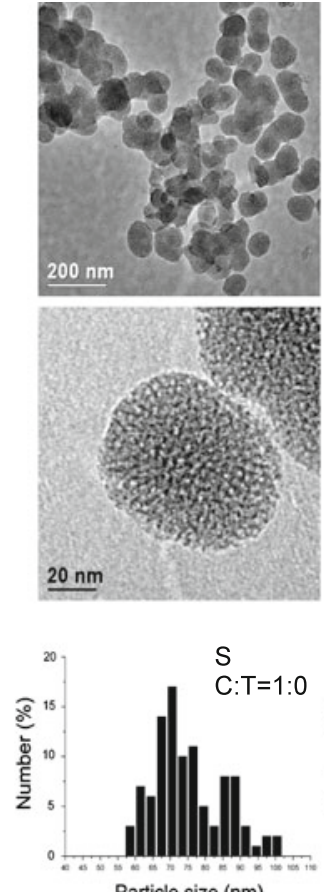

Particle size $(\mathrm{nm})$
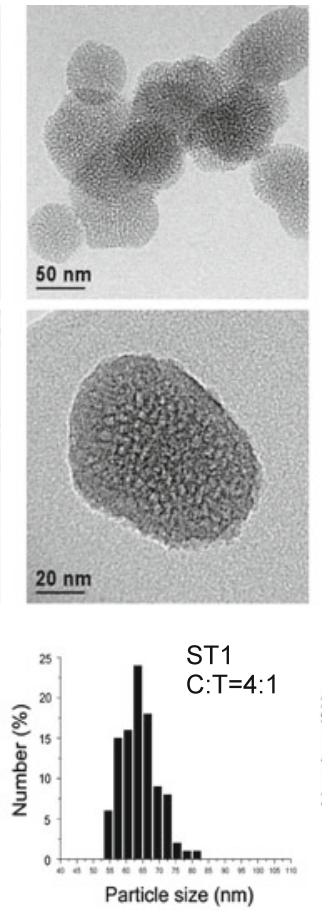
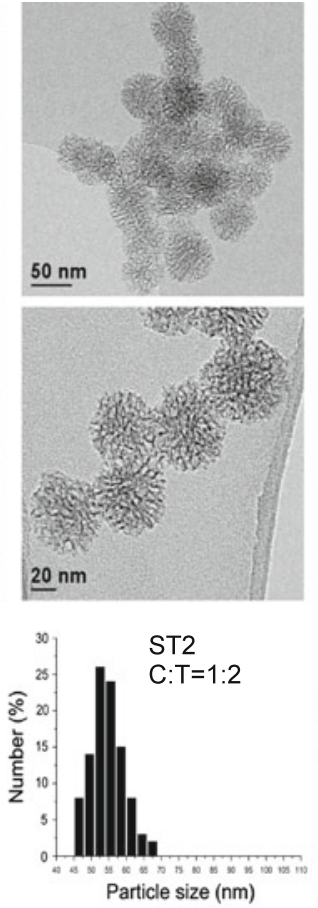
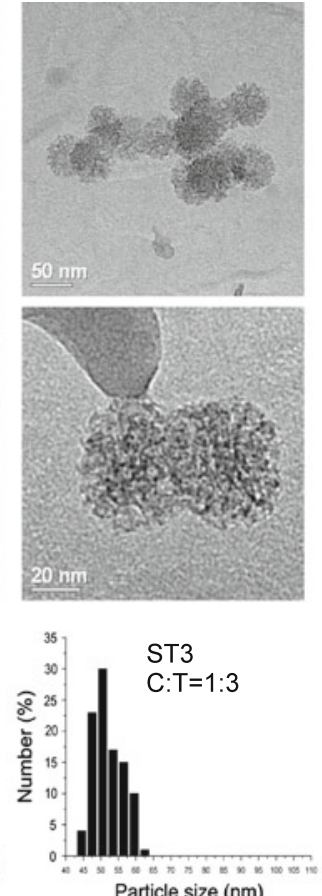

Particle size (nm)
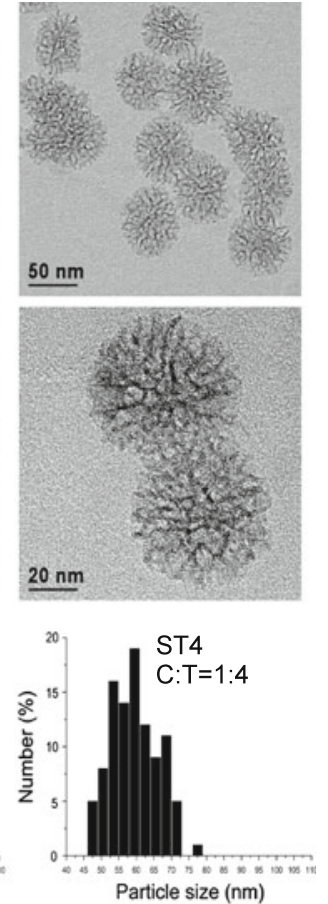

Fig. 3 TEM images of the samples S, ST1-4, and particles size distributions 

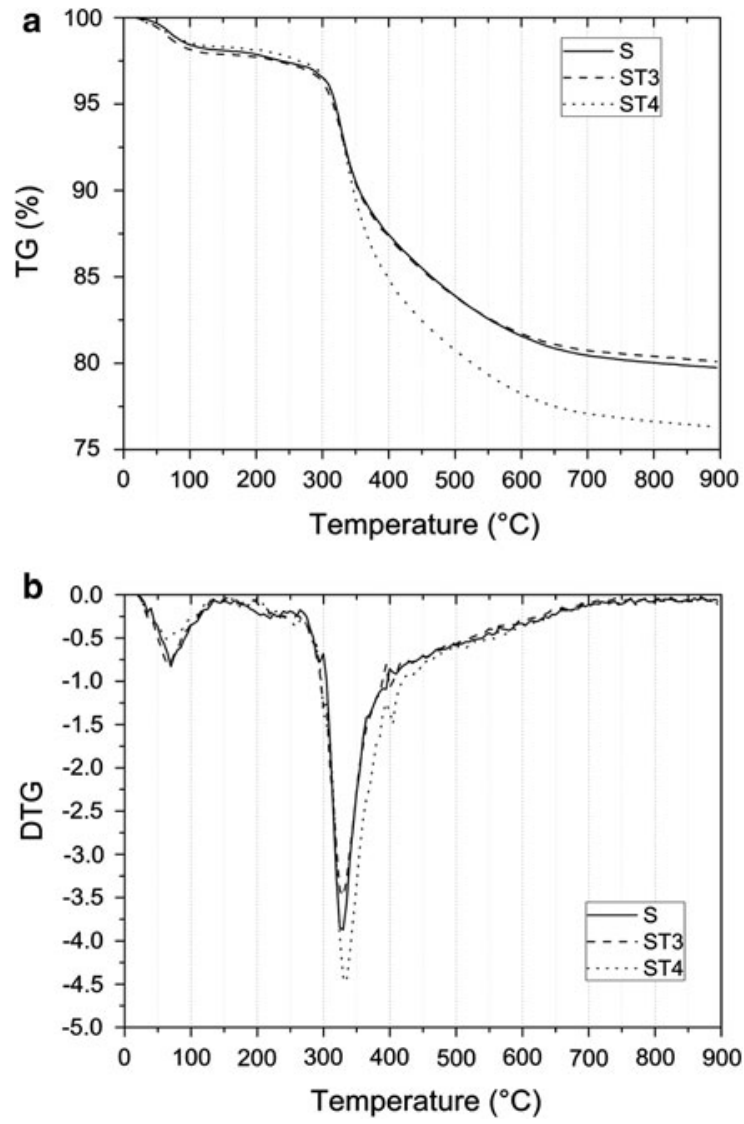

Fig. 4 Thermogravimetric curves (a) and their derivates (b) of sample S, ST3, and ST4 after functionalization with thiol groups

increasingly thin in sample ST4. This trend was also confirmed by the total amount of pore volume. By comparing the morphologies of the samples there is a tendency to form roundish particles with decreasing C:T molar ratio, while the dimension of the particles was relatively constant showing a 50-70-nm diameter (see Table 1). The oscillations of the mean value can be explained considering the irregular porous contours. While samples S and ST1 showed a high degree of agglomeration, the presence of higher quantities of toluene clearly lowered the degree of agglomeration. The diameter of every particle was measured twice, along different directional axis in the pictures. It could be appreciated a good and definite size distribution of the particles for almost all samples. In Fig. S5, images of sample ST5 were presented. In this sample neither a regular shape nor a size distributions were identifiable. The small pore channels were also difficult to detect.
Stabilization of AuNPs in MSNs

Samples S, ST3 and ST4, with three different sizes of pore diameter (see Table 1), were used as templates for internal growth of AuNPs. The pore size and the superficial $-\mathrm{OH}$ groups of the selected silica, allowed the surface functionalization by post grafting the silanol group with (3-mercaptopropyl)trimethoxysilane (MPTS). TGA of the Au-mercaptopropyl-MSN, reported in Fig. 4, demonstrated that the different pore size of the selected materials did not influence the temperature of thiol groups degradation which occurred at about $330{ }^{\circ} \mathrm{C}$ in all samples, in agreement with literature (Díaz et al. 2003).

High resolution TEM images in Fig. 5 of the asprepared Au-thiol-MSNs clearly show that the functionalization of the silica surface give rise to a quite narrow distribution of the size of the gold nanoparticles anchored to the MSNs (Fig. 5a, d, g).

In order to follow the evolution of the metal nanoparticles with increasing temperatures suitable in situ WAXS measurements were collected with a specially designed furnace at the materials characterization by X-ray diffraction beam line at Elettra (Riello et al. 2013). The apparatus provides atmosphere and temperature controlled environment for powders contained in capillaries. The WAXS measurements were collected from room temperature (RT) to $500{ }^{\circ} \mathrm{C}$.

In the spectra, reported in SI (Fig. S6), the intense background from 0.3 to $0.8 \AA^{-1}$ and the peak at $0.25 \AA^{-1}$ were characteristic of both amorphous silica (constituting the MSNs) and of the quartz capillary used as sample holder. Even if in the samples $\mathrm{Au} @ \mathrm{~S}$ and Au@ST3 the presence of very weak peaks was visible at $250{ }^{\circ} \mathrm{C}$ (especially in sample Au@ST3) a fast growth of the gold peaks starts at $350{ }^{\circ} \mathrm{C}$. In any case, in these samples, the peaks characteristic of the gold metallic phase were visible only after a suitable thermal treatment. The absence of diffraction peaks in XRPD spectra, up to $200{ }^{\circ} \mathrm{C}$, for the Au@S and Au@ST3 samples, can be explained assuming that, at lower temperature, the visible particles by TEM (Fig. 5a, d) were probably too small, very disordered or not completely reduced to give a perceptible diffraction peak. In fact, the presence of chlorine into the structure of the as-prepared samples could not be excluded as reported by the SEM-EDS analyses (Fig. 6). The same figure shows that at $350{ }^{\circ} \mathrm{C}$ 

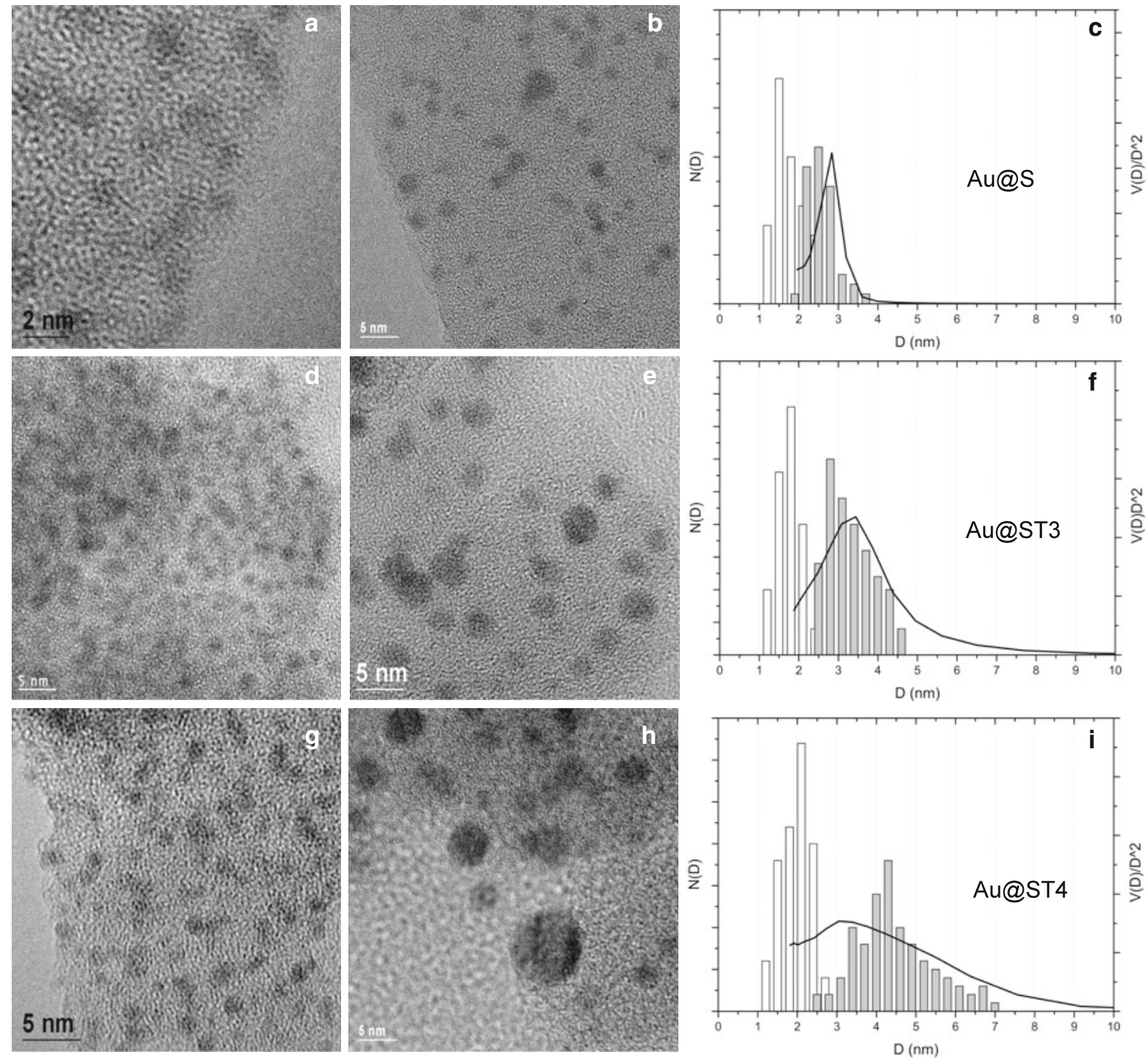

Fig. 5 High resolution TEM images of samples S, ST3, and ST4 with AuNPs grown into their porous network before (a, d, g) and after $(\mathbf{b}, \mathbf{e}, \mathbf{h})$ thermal treatment at $350{ }^{\circ} \mathrm{C}$ with relative

chlorine is no more detectable, as well as sulfur, as expected by the TGA analysis.

On the contrary, XRPD profile of the sample Au@ST4 shows gold crystalline peaks even before the thermal treatment. As it can be seen by TEM of Fig. S7, the XRPD peaks of Au@ST4 sample, at low temperature, were due to the presence of some large AuNPs outside the pore network. This happens even if the silica has been functionalized due to the large poresize distribution of the host ST4 material; in fact, the gold species can migrate to some extent also to the particle-size distribution (white histogram before and black histogram after) comparing with the pore distribution (dotted curves) (c, f, i)

external surface of the MSNs. In this sample it is difficult to identify a well defined threshold temperature for the sintering of the gold particles. In fact, at $200{ }^{\circ} \mathrm{C}$ there is an evident growth of the (200) peak that can be explained with the coalescence of a large amount of hidden particles, not detectable by XRPD at lower temperatures, and with the reduction of the disorder of the large particles out of the pores.

The analysis of the previous results seems to support the idea that thiol groups, which were mandatory for the impregnation of the silica with the 


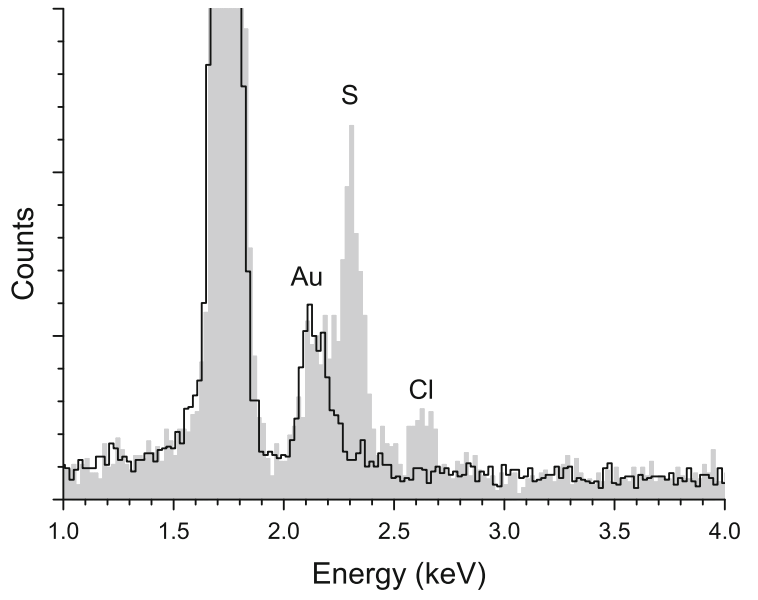

Fig. 6 EDS analysis on Au@MSNs before (gray histogram) and after (black line) thermal treatment at $350{ }^{\circ} \mathrm{C}$ (the profile are similar forAu@S,Au@ST3, and Au@ST4)

gold precursor, are essential during the thermal treatment because they allow the growing of AuNPs inside the silica pore network. Consequently, the observed fast growth of the gold particles at temperature higher than $350{ }^{\circ} \mathrm{C}$ could be correlated with the definitive total degradation of the thiols occurred above this temperature. Only at higher temperatures, the AuNPs size became larger than the pore size and can be expelled from the mesoporous silica structure growing in an uncontrolled way. In order to verify this hypothesis, the samples Au@S, Au@ST3, and Au@ST4 were treated at $350{ }^{\circ} \mathrm{C}$ for $4 \mathrm{~h}$ and analyzed by TEM and ex situ XRPD patterns. The results are reported in Table 2.

The high resolution TEM pictures (Fig. 5b, e, h) and the histograms (Fig. 5c, f, i) show a comparison between Au particle diameter distribution and poresize BJH distribution of the host materials. In order to compare the size distribution of the AuNPs obtain by TEM and the pore-size distribution obtain by $\mathrm{BJH}$ method (see Fig. 2) its necessary take into account the different definition of the two distributions. TEM distribution $N(D) \mathrm{d} D$ is "numerical" one, i.e., is proportional to the number of particles with diameter $D$ in the range $(D \div D+\mathrm{d} D)$; while the distribution $V(D) \mathrm{d} D$ obtained by BJH method is a "volumetric" one, i.e., is proportional to the volume of pores with diameter in the range $(D \div D+\mathrm{d} D)$. Assuming that the average pore length $L$ is independent of the pore diameter the two distribution are related by the equation:

$V(D) \mathrm{d} D=N(D) \frac{\pi D^{2}}{4} L \mathrm{~d} D$.

In Fig. $5 \mathrm{c}, \mathrm{f}$, i, we compare the $N(D)$ obtain by TEM with $V(D) / D^{2}$ obtain by BJH. Au particles sizes really seem to depend on the microstructure of the MSNs and are strictly related to the size of the silica pores. This is an important evidence of the MSNs capacity to stabilize the dimensional growth of nanomaterials. As a matter of fact the movements of the particles are more difficult in a smaller and restricted space than in an opened one.

Moreover, Fig. 7 shows the XRPD patterns of the samples treated at $350{ }^{\circ} \mathrm{C}$. A quantitative analysis of the gold content in all the samples was obtained by a standard approach based on the Rietveld refinement of the diffraction data (Riello et al. 1998a). The synchrotron measurements were not suitable to this aim since the silica contribution of MSNs cannot be evidenced from the quartz glass of the sample holder. The results, reported in Table 2 , are very close to the nominal loading of the sample $(8 \mathrm{wt} \%)$ and they confirm a correlation between the mesoporous silica structure and the size of the metallic particles. Diffraction peaks of the sample Au@ST4 are less broadened than what is expected from the size of the particles that result from the TEM but, as just

Table 2 Summary of AuNPs data derived from TEM and XRPD measurements

\begin{tabular}{llllll}
\hline Sample & $\begin{array}{l}\text { BJH pore } \\
\text { diameter }(\mathrm{nm})\end{array}$ & $\begin{array}{l}\text { TEM particles size } \\
\text { before treatment }(\mathrm{nm})\end{array}$ & $\begin{array}{l}\text { TEM particles size } \\
\text { after treatment }(\mathrm{nm})\end{array}$ & $\begin{array}{l}\text { XRPD particles size } \\
\text { after treatment (nm) }\end{array}$ & $\begin{array}{l}\text { XRPD Au } \\
\text { fraction (wt\%) }\end{array}$ \\
\hline Au@S & 2.8 & $1.7 \pm 0.3$ & $2.5 \pm 0.4$ & 2.0 & 8.3 \\
Au@ST3 & 3.4 & $1.8 \pm 0.3$ & $3.2 \pm 0.6$ & 2.5 & 8.2 \\
Au@ST4 & 5.5 & $2.0 \pm 0.4$ & $4.8 \pm 1.1$ & 5.3 & $7.9^{\mathrm{a}}$ \\
\hline
\end{tabular}

The standard deviation for XRPD particles size distribution is about $10 \%$

a The $42 \%$ of the global loading is given by the particles inside the pores 


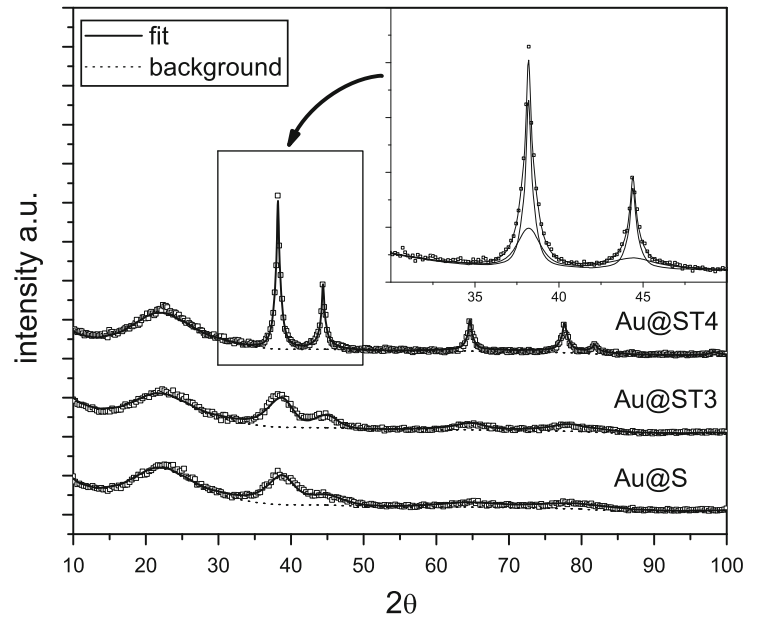

Fig. 7 XRPD patterns of sample Au@S, Au@ST3, and $\mathrm{Au} @ \mathrm{ST} 4$ after treatment at $350{ }^{\circ} \mathrm{C}$

discussed, some larger particles were also present in the sample before heat treatment (Fig. S7). However, in order to obtain the total gold fraction (wt\%), reported in Table 2, it is necessary to consider two groups of particles with very different sizes. This procedure has been already successfully used for a $\mathrm{Pd} / \mathrm{SiO}_{2}$ and $\mathrm{Au} / \mathrm{C}$ systems to evidence and to quantify the presence of two populations of particles with a large difference of size (Polizzi et al. 2001; Riello et al. 1998b). Following this procedure two different two peaks with very different half width were necessary to properly describe the line profile (see inset in Fig. 7). The results, relative to the particles with smaller size and reported in Table 2, still confirm the good correlation between the mesoporous structure and the size of the AuNPs.

\section{Conclusions}

The main aim of the paper concerns the preparation of carriers based on silica nanoparticles (MSNs) with a controlled mesoporous structure, in order to develop multi-use systems for different applications such as adsorption, sensing, catalysis, separation, and nanomedicine. A correlation among the average pore size of the mesoporous silica nanoparticles and the final growth of AuNPs within the silica pores has been investigated. A simple method for the synthesis of MSNs and their pore size tuning was presented. Ordered MSNs, with different pore size were obtained via a surfactant-assisted synthesis using $\mathrm{CTAB}$ and toluene as micelle core-swelling agent. BET, SAXS, and TEM analyses confirmed that the largest pores were achieved with a CTAB:toluene molar ratio of 1:4. The thermal treatment, needed to remove the template, produces superficial silanol groups, thus allowing MSNs thiol functionalization. The growth of the AuNPs starts when the temperature of the thermal treatment of the $\mathrm{HAuCl}_{4}$ impregnated MSNs reaches the degradation temperature of the thiol groups $\left(350{ }^{\circ} \mathrm{C}\right)$. The size of the AuNPs in this condition is controlled by the pore diameter of the MSNs. A fast growth of the gold particles outside the mesoporous structure has been observed at higher temperatures. In any case the presence of thiol groups was found to be indispensable to allow the growth of AuNPs inside the silica pore network. The possibility to control the size and the amount of a single phase, with a specific functionality, loaded into the mesoporous structure can be an important step for further developments of tailored nanosystems. In this case the gold particles are stabilized by the pore networks that prevent the agglomeration of the metallic nanoclusters without any surface functionalization.

Acknowledgements The authors thank Mr D. Cristofori for bare silica TEM images, Mr T. Finotto for the SAXS and XRPD measurements and Mr. N. Mazzucco for EDS analysis.

\section{References}

Asaro F, Benedetti A, Freris I, Riello P, Savko N (2010) Evolution of the nonionic inverse microemulsion-acid-TEOS system during the synthesis of nanosized silica via the solgel process. Langmuir 26:12917-12925. doi:10.1021/ la101737x

Badaničová M, Zeleňák V (2010) Organo-modified mesoporous silica for sorption of carbon dioxide. Monatsh Chem 141:677-684. doi:10.1007/s00706-010-0304-6

Bae JY, Choi S, Bae B (2006) Preparation and optical characterization of mesoporous silica films with different pore sizes. Bull Korean Chem Soc 27:1562-1566

Beck JS, Vartuli JC, Roth WJ, Leonowicz ME, Kresge CT, Schmitt KD, Chu CTW, Olson DH, Sheppard EW (1992) A new family of mesoporous molecular sieves prepared with liquid crystal templates. J Am Chem Soc 114:1083410843. doi:10.1021/ja00053a020

Bergman L, Kankaanpää P, Tiitta S, Duchanoy A, Li L, Heino J, Lindén M (2013) Intracellular degradation of multilabeled poly(ethylene imine)-mesoporous silica-silica nanoparticles: implications for drug release. Mol Pharm 10:1795-1803. doi:10.1021/mp3005879 
Biz S, Occelli M (1998) Synthesis and characterization of mesostructured materials. Catal Rev 40:329-407. doi:10. 1080/01614949808007111

Büchel G, Grün M, Unger KK, Matsumoto A (1998) Tailored syntheses of nanostructured silicas: control of particle morphology, particle size and pore size. Supramol Sci 5:253-259. doi:10.1016/S0968-5677(98)00016-9

Cai W, Gao T, Sun J (2008) Applications of gold nanoparticles in cancer nanotechnology. Nanotechnol Sci Appl 1:17-32. doi:10.2147/NSA.S3788

Cao L, Kruk M (2010) Synthesis of large-pore SBA-15 silica from tetramethyl orthosilicate using triisopropylbenzene as micelle expander. Colloids Surf A 357:91-96. doi:10. 1016/j.colsurfa.2009.09.019

Cauda V, Argyo C, Bein T (2010a) Impact of different PEGylation patterns on the long-term bio-stability of colloidal mesoporous silica nanoparticles. J Mater Chem 20:8693-8699. doi:10.1039/c0jm01390k

Cauda V, Scholossbauer A, Bein T (2010b) Bio-degradation study of colloidal mesoporous silica nanoparticles: effect of surface functionalization with organo-silanes and poly(ethylene glycol). Microporous and Mesoporous Mater 132:60-71. doi:10.1016/j.micromeso.2009.11.015

Chen TM, Brauer GM (1982) Solvent effects on bonding organo-silane to silica surfaces. J Dent Res 61:1439-1443. doi:10.1177/00220345820610121301

Choma J, Dziura A, Jamiola D, Nyga P, Jaroniec M (2011) Preparation and properties of silica-gold core-shell particles. Coll Surf A 373:167-171. doi:10.1016/j.colsurfa. 2010.10.046

Choma J, Dziura A, Jamiola D, Nyga P, Jaroniec M (2012) Deposition of silver nanoparticles on silica spheres and rods. Coll Surf A 411:74-79. doi:10.1016/j.colsurfa.2012.07.004

Corma A, Garcia H (2008) Supported gold nanoparticles as catalysts for organic reactions. Chem Soc Rev 27:2096-2126. doi:10.1039/B707314N

Cuenya BR (2010) Synthesis and catalytic properties of metal nanoparticles: size, shape, support, composition, and oxidation state effects. Thin Solid Films 518:3127-3150. doi:10.1016/j.tsf.2010.01.018

de Dios AS, Díaz-García ME (2010) Multifunctional nanoparticles: analytical prospects. Anal Chim Acta 666:1-22. doi:10.1016/j.aca.2010.03.038

Díaz I, Mohino F, Pérez-Pariente J, Sastre E (2003) Synthesis of MCM-41 materials functionalised with dialkylsilane groups and their catalytic activity in the esterification of glycerol with fatty acids. Appl Catal A 242:161-169. doi:10.1016/S0926-860X(02)00501-X

Enrichi F, Riccò R, Meneghello A, Pierobon R, Cretaio E, Marinello F, Schiavuta P, Parma A, Riello P, Benedetti A (2010) Investigation of luminescent dye-doped or rareearth-doped monodisperse silica nanospheres for DNA microarray labelling. Opt Mater 32:1652-1658. doi:10. 1016/j.optmat.2010.04.026

Fan J, Yu C, Gao F, Lei J, Tian B, Wang L, Luo Q, Tu B, Zhou W, Zhao D (2003) Cubic mesoporous silica with large controllable entrance sizes and advanced adsorption properties. Angew Chem 115:3254-3258. doi:10.1002/ ange. 200351027

Fenger R, Fertitta E, Kirmse H, Thünemann AF, Rademann K (2012) Size dependent catalysis with CTAB-stabilized gold nanoparticles. Phys Chem Chem Phys 14:9343-9349. doi:10.1039/c2cp40792b10.1039/c2cp40792b

Firouzi A, Kumar D, Bull LM, Besier T, Sieger P, Huo Q, Walker SA, Zasadzinski JA, Glinka C, Nicol J (1995) Cooperative organization of inorganic-surfactant and biomimetic assemblies. Science 267:1138-1143. doi:10.1126/ science. 7855591

Fuertes AB, Valle-Vigón P, Sevilla M (2010) Synthesis of colloidal silica nanoparticles of a tunable mesopore size and their application to the adsorption of biomolecules. J Colloid Interf Sci 349:173-180. doi:10.1016/j.jcis.2010. 05.041

Galarneau A, Iapichella J, Bonhomme K, Di Renzo F, Kooyman P, Terasaki O, Fajula F (2006) Controlling the morphology of mesostructured silicas by pseudomorphic transformation: a route towards applications. Adv Funct Mater 4(16):1657-1667. doi:10.1002/adfm.200500825

Giersig M, Mulvaney P (1993) Preparation of ordered colloid monolayers by electrophoretic deposition. Langmuir 9:3408-3413. doi:10.1021/la00036a014

Hasan M, Bethell D, Brust M (2002) The fate of sulfur-bound hydrogen on formation of self-assembled thiol monolayers on gold: 1H NMR spectroscopic evidence from solutions of gold clusters. J Am Chem Soc 124:1132-1133. doi:10. 1021/ja0120577

He Q, Shi J (2011a) Mesoporous silica nanoparticle based nano drug delivery systems: synthesis, controlled drug release and delivery, pharmacokinetics and biocompatibility. J Mater Chem 21:5845-5855. doi:10.1039/c0jm03851b

He Q, Zhang J, Shi J, Zhu Z, Zhang L, Bu W, Guo L, Chen Y (2010) The effect of PEGylation of mesoporous silica nanoparticles on nonspecific binding of serum proteins and cellular responses. Biomaterials 31:1085-1092. doi:10. 1016/j.biomaterials.2009.10.046

He Q, Shi J, Cui X, Wei C, Zhang L, Wu W, Bu W, Chen H, Wu H (2011b) Synthesis of oxygen-deficient luminescent mesoporous silica nanoparticles for synchronous drug delivery and imaging. Chem Commun 47:7947-7949. doi:10.1039/c1cc11479d

Hoshikawa Y, Yabe H, Nomura A, Yamaki T, Shimojima A, Okubo T (2010) Mesoporous silica nanoparticles with remarkable stability and dispersibility for antireflective coatings. Chem Mater 22:12-14. doi:10.1021/cm902239a

Huang L, Yan X, Kruk M (2010) Synthesis of ultralarge-pore FDU-12 silica with face-centered cubic structure. Langmuir 26:14871-14878. doi:10.1021/la102228u

Huang X, Li L, Liu T, Hao N, Liu H, Chen D, Tang F (2011) The shape effect of mesoporous silica nanoparticles on biodistribution, clearance, and biocompatibility in vivo. ACS Nano 5:5390-5399. doi:10.1021/nn200365a

Jankiewicz BJ, Jamiola D, Choma J, Jaroniec M (2012) Silicametal core-shell nanostructures. Adv Colloid Interface Sci 170:28-49. doi:10.1016/j.cis.2011.11.002

Kim TW, Chung PW, Lin VSY (2010) Facile synthesis of monodisperse spherical MCM-48 mesoporous silica nanoparticles with controlled particle size. Chem Mater 22:5093-5104. doi:10.1021/cm1017344

Krawiec P, Kockrick E, Simon P, Auffermann G, Kaskel S (2006) Platinum-catalyzed template removal for the in situ synthesis of MCM-41 supported catalysts. Chem Mater 18:2663-2669. doi:10.1021/cm052830n 
Kresge CT, Leonowicz ME, Roth WJ, Vartuli JC (1992) Ordered mesoporous molecular sieves synthesized by a liquid-crystal template mechanism. Nature 359:710-712. doi: $10.1038 / 359710 \mathrm{a} 0$

Kruk M, Cao L (2007) Pore size tailoring in large-pore SBA-15 silica synthesized in the presence of hexane. Langmuir 23:7247-7254. doi:10.1021/la0702178

Kruk M, Jaroniec M, Sayari A (2000) New insights into poresize expansion of mesoporous silicates using long-chain amines. Microporous and Mesoporous Mater 35(36): 545-553. doi:10.1016/S1387-1811(99)00249-8

Lai CY, Trewyn BG, Jeftinija DM, Jeftinija K, Xu S, Jeftinija S, Lin VSY (2003) A mesoporous silica nanosphere-based carrier system with chemically removable CdS nanoparticle caps for stimuli-responsive controlled release of neurotransmitters and drug molecules. J Am Chem Soc 125:4451-4459. doi:10.1021/ja0286501

Lee B, Ma Z, Zhang Z, Park C, Dai S (2009) Influences of synthesis conditions and mesoporous structures on the gold nanoparticles supported on mesoporous silica hosts. Microporous and Mesoporous Mater 122:160-167. doi:10. 1016/j.micromeso.2009.02.029

Lefèvre B, Galarneau A, Iapichella J, Petitto C, Di Renzo F, Fajula F, Bayram-Hahn Z, Skudas R, Unger K (2005) Synthesis of large-pore mesostructured micelle-templated silicas as discrete spheres. Chem Mater 17:601-607. doi: $10.1021 / \mathrm{cm} 048481 \mathrm{z}$

Li ZH, Gong YJ, Wu D, Sun YH, Wang J, Liu Y, Dong BZ (2001) SAXS analysis of interface in organo-modified mesoporous silica. Surf Interface Anal 31:897-900. doi:10. 1002/sia.1118

Lin YS, Haynes CL (2009) Anticancer drug release from a mesoporous silica based nanophotocage regulated by either a one- or two-photon process. Chem Mater 21:3979-3986. doi: $10.1021 / \mathrm{cm} 901259 \mathrm{n}$

Lin YS, Haynes CL (2010) Impacts of mesoporous silica nanoparticle size, pore ordering, and pore integrity on hemolytic activity. J Am Chem Soc 132:4834-4842. doi:10.1021/ja910846q

Lindén M, Ågren P, Karlsson S, Bussian P, Amenitsch H (2000) Solubilization of oil in silicate-surfactant mesostructures. Langmuir 16:5831-5836. doi:10.1021/la991671h

Lindlar B, Kogelbauer A, Kooyman PJ, Prins R (2001) Synthesis of large pore silica with a narrow pore size distribution. Microporous Mesoporous Mater 44(45):89-94. doi:10.1016/S1387-1811(01)00172-X

Ma S, Wang Y, Zhu Y (2011) Condensation enthalpies of $n$ hexane in micelle-templated mesoporous silicas. J Porous Mater 14:279-284. doi:10.1007/s10934-006-9063-5

Maldonado D, Tanchoux N, Trens P, Galarneau A, Garrone E, Renzo F, Fajula F (2007) A simple room temperature synthesis of mesoporous silica nanoparticles for drug storage and pressure pulsed delivery. J Porous Mater 18:233-239. doi:10.1007/s10934-010-9375-3

Marquez DT, Carrillo AI, Scaiano JC (2013) Plasmon excitation of supported gold nanoparticles can control molecular release from supramolecular systems. Langmuir 29:10521-10528. doi:10.1021/la4019794

Nandiyanto ABD, Kim SG, Iskandar F, Okuyama K (2009) Synthesis of spherical mesoporous silica nanoparticles with nanometer-size controllable pores and outer diameters. Microporous Mesoporous Mater 120:447-453. doi:10.1016/j.micromeso.2008.12.019

Ottaviani MF, Moscatelli A, Desplantier-Giscard D, Di Renzo F, Kooyman PJ, Alonso B, Galarneau A (2004) Synthesis of micelle-templated silicas from cetyltrimethylammonium bromide/1,3,5-trimethylbenzene micelles. J Phys Chem B 108:12123-12129. doi:10.1021/jp049032p

Pan Y, Neuss S, Leifert A, Fischler M, Wen F, Simon U, Schmid G, Brandau W, Jahnen-Dechent W (2007) Size-dependent cytotoxicity of gold nanoparticles. Small 3:1941-1949. doi:10.1002/smll.200700378

Park JH, Gu L, Von Maltzahn G, Ruoslahti E, Bhatia SN, Sailor MJ (2009) Biodegradable luminescent porous silicon nanoparticles for in vivo applications. Nat Mater 8:331336. doi:10.1038/nmat2398

Park HS, Kim CW, Lee HJ, Choi JH, Lee SG, Yun YP, Kwon IC, Lee SJ, Jeong SY, Lee SC (2010) A mesoporous silica nanoparticle with charge-convertible pore walls for efficient intracellular protein delivery. Nanotechnology 22:225101. doi:10.1088/0957-4484/21/22/225101

Parma A, Freris I, Riello P, Enrichi F, Cristofori D, Benedetti A (2010) Structural and photoluminescence properties of $\mathrm{ZrO}_{2}: \mathrm{Eu}^{3+} @ \mathrm{SiO}_{2}$ nanophosphors as a function of annealing temperature. J Lumin 130:2429-2436. doi:10.1016/j. jlumin.2010.08.007

Polizzi S, Riello P, Balerna A, Benedetti A (2001) Nanostructure of $\mathrm{Pd} / \mathrm{SiO}_{2}$ supported catalysts. Phys Chem Chem Phys 3:4614-4619. doi:10.1039/b105607g

Poteshnova MV, Zadymova NM (2006) Normal micelles and oil-in-water microemulsions in a water-toluene-tween 80 ternary system. Colloid J 68:201-210. doi:10.1134/S1061 933X06020128

Qiao ZA, Zhang L, Guo M, Liu Y, Huo Q (2009) Synthesis of mesoporous silica nanoparticles via controlled hydrolysis and condensation of silicon alkoxide. Chem Mater 21:3823-3829. doi:10.1021/cm901335k

Riello P, Canton P, Fagherazzi G (1998a) Quantitative phase analysis in semicrystalline materials using the rietveld method. J App Cryst 31:78-82. doi:10.1107/S002188989 7009631

Riello P, Canton P, Benedetti A (1998b) Au/C catalyst: experimental evidence of the coexistence of nanoclusters and larger Au particles. Langmuir 14:6617-6619. doi:10.1021/ la9803272

Riello P, Lausi A, Macleaod J, Plaisier JR, Zeraushek G, Fornasiero $P$ (2013) In situ reaction furnace for real-time XRD studies. J Synchrotron Rad 20:194-196. doi:10.1107/ S0909049512039246

Saha K, Agasti SS, Kim C, Li X, Rotello VM (2012) Gold nanoparticles in chemical and biological sensing. Chem Rev 112:2739-2779. doi:10.1021/cr2001178

Scaffardi LB, Tocho JO (2006) Size dependence of refractive index of gold nanoparticles. Nanotechnology 17:13091315. doi:10.1088/0957-4484/17/5/024

Schulz-Ekloff G, Rathouskỳ J, Zukal A (1999) Mesoporous silica with controlled porous structure and regular morphology. Int J Inorg Mater 1:97-102. doi:10.1016/S14630176(99)00015-0

Schumacher K, Ravikovitch PI, Du Chesne A, Neimark AV, Unger KK (2000) Characterization of MCM-48 materials. Langmuir 16:4648-4654. doi:10.1021/la991595i 
Sivestrini S, Riello P, Freris I, Cristofori D, Enrichi F, Benedetti A (2010) Structural and luminescence properties of europium(III)-doped zirconium carbonates and silica-supported $\mathrm{Eu}^{3+}$-doped zirconium carbonate nanoparticles. J Nanopart Res 12:993-1002. doi:10.1007/s11051-0099655-5

Storaro L, Lenarda M, Moretti E, Talon A, Porta F, Moltrasio B, Canton P (2010) Gold stabilized aqueous sols immobilized on mesoporous $\mathrm{CeO}_{2}-\mathrm{Al}_{2} \mathrm{O}_{3}$ as catalysts for the preferential oxidation of carbon monoxide. J Colloid Interf Sci 350:435-442. doi:10.1016/j.jcis.2010.06.062

Stratakis M, Garcia H (2012) Catalysis by supported gold nanoparticles: beyond aerobic oxidative processes. Chem Rev 112:4469-4506. doi:10.1021/cr3000785

Trewyn BG, Slowing II, Giri S, Chen HT, Lin VSY (2007) Synthesis and functionalization of a mesoporous silica nanoparticle based on the sol-gel process and applications in controlled release. Accounts Chem Res 40:846-853. doi:10.1021/ar600032u

Upadhyayula VKK (2012) gold nanoparticle supported sensory mechanisms applied in detection of chemical and biological threat agents: a review. Anal Chim Acta 715:1-18. doi:10.1016/j.aca.2011.12.008

Vivero-Escoto JL, Slowing II, Wu CW, Lin VS (2009) Intracellular controlled release drug delivery in human celisby gold-capped mesoporous silica nanosphere. J Am Chem Soc 131:3462-3463. doi:10.1021/ja900025f
Wan Y, Zhao D (2007) On the controllable soft-templating approach to mesoporous silicates. Chem Rev 107:28212860. doi:10.1021/cr068020s

Wu SH, Hung Y, Mou CY (2011) Mesoporous silica nanoparticles as nanocarriers. Chem Commun 47:9972-9985. doi: $10.1039 / \mathrm{C} 1 \mathrm{CC} 11760 \mathrm{~B}$

Yah CS (2013) The toxicity of gold nanoparticles in relation to their physiochemical properties. Biomed Res 24:400-413

Zhao Y, Sun X, Zhang G, Trewyn BG, Slowing II, Lin VSY (2011) Interaction of mesoporous silica nanoparticles with human red blood cell membranes: size and surface effects. ACS Nano 5:1366-1375. doi:10.1021/nn103077k

Zhou W, Hunter HMA, Wright PA, Ge Q, Thomas JM (1998) Imaging the pore structure and polytypic intergrowths in mesoporous silica. J Phys Chem B 102:6933-6936. doi:10. 1021/jp982579h

Zhou X, Xu M, Liu G, Panda D, Chen P (2010) Size-dependent catalytic activity and dynamics of gold nanoparticles at the single-molecule level. J Am Chem Soc 132:138-146. doi: $10.1021 /$ ja904307n

Zong J, Zhu Y, Yang X, Li C (2011) Preparation of monodispersed mesoporous silica spheres with tunable pore size and pore-size effects on adsorption of Au nanoparticles and urease. Mater Sci Eng C 31:166-172. doi:10.1016/j.msec. 2010.08.014 